OPEN ACCESS

Edited by:

Luigi Lavorgna,

University of Campania Luigi

Vanvitelli, Italy

Reviewed by:

Yoshiro Ohara,

Kanazawa Medical University, Japan

Miriam Laura Fichtner,

Yale Medicine, United States

*Correspondence:

Johanna Sjöwall

johanna.sjowall@liu.se

Specialty section:

This article was submitted to

Multiple Sclerosis and

Neuroimmunology,

a section of the journal

Frontiers in Neurology

Received: 22 December 2020

Accepted: 25 January 2021

Published: 12 February 2021

Citation:

Sjöwall J, Xirotagaros $G$, Anderson CD, Sjöwall $C$ and Dahle $C$ (2021) Case Report: Borrelia-DNA Revealed the Cause of Arthritis and Dermatitis During Treatment With Rituximab. Front. Neurol. 12:645298. doi: 10.3389/fneur.2021.645298

\section{Case Report: Borrelia-DNA Revealed the Cause of Arthritis and Dermatitis During Treatment With Rituximab}

\author{
Johanna Sjöwall ${ }^{1 *}$, Georgios Xirotagaros ${ }^{2}$, Chris D. Anderson ${ }^{2}$, Christopher Sjöwall ${ }^{3}$ and \\ Charlotte Dahle ${ }^{4}$
}

${ }^{1}$ Infectious Diseases/Division of Inflammation and Infection, Department of Biomedical and Clinical Sciences, Linköping University, Linköping, Sweden, ${ }^{2}$ Dermatology and Venereology, Department of Biomedical and Clinical Sciences, Linköping University, Linköping, Sweden, ${ }^{3}$ Rheumatology/Division of Inflammation and Infection, Department of Biomedical and Clinical Sciences, Linköping University, Linköping, Sweden, ${ }^{4}$ Clinical Immunology and Transfusion Medicine/Division of Inflammation and Infection, Department of Biomedical and Clinical Sciences, Linköping University, Linköping, Sweden

Borrelia-specific antibodies in serum did not contribute to the diagnosis of Borrelia arthritis or Borrelia-associated dermatitis in a young woman with ongoing treatment with rituximab due to multiple sclerosis. The diagnosis was confirmed by the detection of Borrelia-DNA in a skin punch biopsy. The patient history did not reveal any tick exposure. She had suffered for several months from fluctuating pain and swelling of the right knee as well as skin involvement with redness and oedema around the ankle of the same leg. Monoarthritis was confirmed by a rheumatologist. Knee puncture was performed but the synovial fluid was only sufficient for microscopic examination of crystals. Neither monosodium urate crystals nor calcium pyrophosphate crystals were found. Borrelia serology in blood revealed borderline levels of immunoglobulin (lg)M and lgG, respectively. Treatment with doxycycline resulted in resolution of the joint and skin manifestations within a month. This case highlights that Borrelia-specific antibody levels cannot be reliably interpreted in patients who have received B-cell depleting therapy. Under these circumstances, detection of the bacterial genome in different body fluids, such as in the skin, can be a useful complement to the diagnosis of Lyme disease. In this young female, the diagnosis would certainly have been further delayed without the detection of Borrelia-DNA in the skin.

Keywords: Lyme disease, Borrelia-DNA, arthritis, dermatitis, rituximab, Borrelia serology

\section{INTRODUCTION}

An atypical clinical presentation and absence of an adequate immune response to infections are common phenomena in patients with primary immunodeficiency disorders (PID) (1) and multiple sclerosis (MS) (2), as well as in subjects receiving immunosuppressive agents (3). Furthermore, several immune modulating therapies may have similar effects, but this is less wellrecognized among physicians lacking deeper knowledge of the impact of the immune system on the pathogenesis and the clinical manifestations of different infectious diseases. As immunomodulating therapies (IMT) are now widely used for a variety of autoimmune and inflammatory diseases, 
specialists of many different disciplines may need to treat patients using this group of drugs. For instance, patients with MS are often diagnosed and started on treatment at young age and continue for years or decades (4). These circumstances emphasize the importance of physicians being aware of atypical reactions regarding both clinical symptoms and laboratory test results obscuring infections among these patients. This case illustrates that an ongoing infection can easily be overlooked or misinterpreted due to a weak serological response during treatment with a B-cell depleting drug.

\section{CASE PRESENTATION}

This case illustrates a 20-year-old female diagnosed with MS at the age of 17 . She was initially treated with tocilizumab as neuromyelitis optica was suspected due to bilateral optical neuritis and the presence of spinal cord lesions. However, antibodies against aquaporin-4 and myelin oligodendrocyte glycoprotein were not detected and the magnetic resonance imaging (MRI) of the brain and spinal cord as well as cerebrospinal fluid (CSF) findings were supportive of MS. Apart from persistent bilateral severely reduced visual acuity she had no other signs of neurological dysfunction. She had previously been in good health and had no family history of PID, or other systemic inflammatory diseases.

Eighteen months prior to the episode of arthritis and skin symptoms reported here, she was started on off-label treatment with rituximab (RTX). RTX is the most frequently used immunomodulatory drug for MS in Sweden according to the Swedish MS registry (5). Initially, she received $1,000 \mathrm{mg}$ of RTX followed by $500-1,000 \mathrm{mg}$ every 6th month, resulting in depletion of circulating B-cells $\left(<0.001 \times 10^{9} / \mathrm{L}\right)$. During this period, there were no signs of neuroinflammatory activity of MS.

\section{Clinical Episode}

A rheumatologist confirmed the diagnosis of monoarthritis. The right knee had typical signs of inflammation with rubor, tumor, and calor accompanied by a discretely reduced range of motion. The general status was good without fever. The lower right leg was diffusely swollen and two circular erythematous areas around the ankle were seen (Figures 1A,B). A dermatologist interpreted the skin symptoms as possible panniculitis with atypical erythema nodosum as a potential alternative diagnosis. There were no other clinical or laboratory findings of sarcoidosis.

\section{Timeline}

Treatment with RTX had been ongoing for approximately one and a half year prior to the onset of arthritis and the cutaneous symptoms had been present for at least 6 months prior to the diagnosis. The last dose of RTX was given 1.5 months before the onset of symptoms related to Lyme disease.

\section{Diagnostic Assessments}

Aspiration of synovial fluid resulted in a limited volume, only sufficient for microscopic examination of crystals. Neither monosodium urate crystals nor calcium pyrophosphate crystals
A

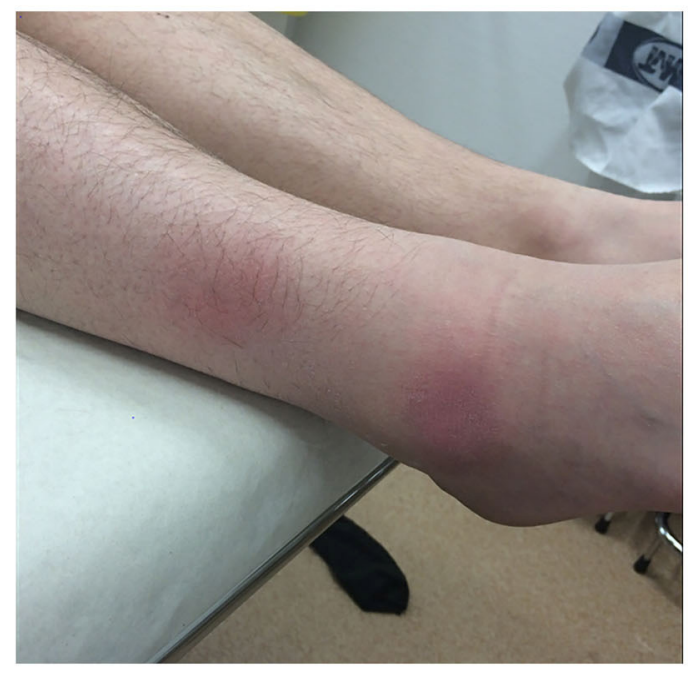

B

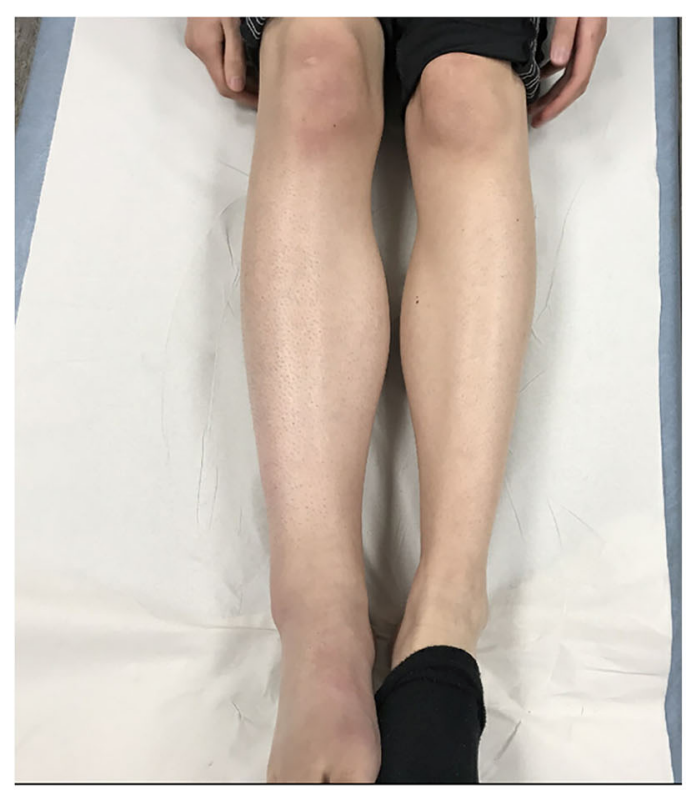

FIGURE 1 | Periarticular swelling of the right leg and ankle. The skin is slightly atrophic adjacent to the two erythematous circular areas seen on the lateral side. The blood vessels appear prominently over the apical part of the foot; a common phenomenon in late cutaneous borreliosis (A). The right knee, calf, and ankle are swollen, without a distinct erythema. Fifteen to twenty degrees deficit in knee extension was observed. Note the dark discoloration of the medial and apical parts of the foot, typically seen in patients with late cutaneous borreliosis (B) 
were detected in the joint fluid. Duplex ultrasonography of the lower leg showed no signs of deep vein thrombosis and there were no laboratory signs of systemic inflammation. Serological analysis performed 5 months after the last dose of RTX showed borderline levels of immunoglobulin (Ig)M and IgG antibodies against recombinant Borrelia antigens (Liason ${ }^{\circledR}$, Borrelia IgM detecting OspC and VlsE; Borrelia IgG detecting VlsE). The results were interpreted to be of uncertain clinical significance. Laboratory results are detailed in Table 1.

Despite the vague antibody results, there were an enduring clinical suspicion of Borrelia infection. Skin biopsies from one of the erythematous areas at the ankle were performed. Standard histopathology showed mild non-specific inflammation. Borrelia-DNA was detected in the biopsy analyzed by polymerase chain reaction (PCR). The method amplifies a 116 base-pair long fragment of the 16S rRNA gene. In addition, a lumbar puncture was done, and CSF was analyzed without presence of intrathecal Borrelia-specific antibodies or elevated levels of the B-cell chemokine CXCL13. Thus, the final diagnosis was Borrelia associated dermatitis and arthritis (Lyme disease).

\section{Therapeutic Intervention}

Prior to the diagnosis of Lyme disease, the patient was prescribed topical steroids for the skin manifestations and the joint symptoms were managed with paracetamol. Once the diagnosis of Lyme disease was confirmed, doxycycline $200 \mathrm{mg}$ once daily for 3 weeks was prescribed. The knee and skin symptoms dissipated during the following month.

\section{Follow-Up and Outcome}

At the last follow-up 1 year after the antibiotic treatment had been ended, there was still minor swelling of the lower leg but no signs of arthritis or dermatitis. An MRI of the lower leg showed mild oedema in musculus soleus and gastrocnemius. Creatinine kinase in plasma was within normal reference.

\section{DISCUSSION}

IMT in general, and particularly B-cell depleting therapies, may be associated with an increased risk of infections $(2,6,7)$. Serological screening for IgG against several infectious agents is therefore routinely performed prior to initiation of IMT and vaccination should be considered when immunity is not detected. However, the fact that IMT can have an impact on the clinical picture and serological response to infectious agents is less well recognized among physicians outside the field of immunology and infectious diseases. B-cell depleting therapies are widely used in MS as well as in many other autoimmune diseases, often with a dramatic anti-inflammatory effect and symptom relief (6). In chronic inflammatory diseases like MS, the treatment is often continued for many years and results in undetectable or very low numbers of circulating B-cells. Although RTX, compared to other disease modifying drugs in MS, has been shown to be associated with an increased risk for serious infections it is widely used due its marked effect on the disease activity and disease progression (4). Another side effect is weak and non-protective responses
TABLE 1 | Laboratory findings in blood.

\begin{tabular}{lcc}
\hline Analyte & Results & Reference interval \\
\hline Hemoglobin & 151 & $117-153 \mathrm{~g} / \mathrm{L}$ \\
Leukocyte count & 7.2 & $3.5-8.8 \times 10^{9} / \mathrm{L}$ \\
Lymphocyte count & 1.3 & $1.1-4.8 \times 10^{9} / \mathrm{L}$ \\
B-cells & $<0.001$ & $0.075-0.53 \times 10^{9} / \mathrm{L}$ \\
Platelet count & 283 & $160-390 \times 10^{9} / \mathrm{L}$ \\
Erythrocyte sedimentation rate (ESR) & 2 & $<21 \mathrm{~mm} / \mathrm{h}$ \\
P-C-reactive protein (CRP) & $<5$ & $<10 \mathrm{mg} / \mathrm{L}$ \\
S-Creatinine kinase & 1.5 & $<3.6 \mu \mathrm{kat} / \mathrm{L}$ \\
P-Alanine transaminase & 0.35 & $<0.76 \mu \mathrm{kat} / \mathrm{L}$ \\
P-Creatinine & 81 & $45-90 \mu \mathrm{mol} / \mathrm{L}$ \\
P-Urate & 209 & $155-350 \mu \mathrm{mol} / \mathrm{L}$ \\
S-Angiotensin converting enzyme & 0.51 & $<1.1 \mu \mathrm{kat} / \mathrm{L}$ \\
Anti-cyclic citrullinated peptide antibody (lgG) & 1 & $<7 \mathrm{U} / \mathrm{L}$ \\
Borrelia antibody (lgM) & 38.6 & $<30 \mathrm{AU} / \mathrm{mL}$ \\
Borrelia antibody (lgG) & 16.6 & $<10 \mathrm{AU} / \mathrm{mL}$ \\
& &
\end{tabular}

$A U$, arbitrary units; $P$-, analysis in plasma; $S$-, analysis in serum; $U$, units.

to vaccinations, as long as the circulating B-cells are very low $(8,9)$.

In the patient described here, Borrelia caused late skin and joint manifestations that did not raise the clinical suspicion of Lyme disease, until the weak serological response was received. Still, the antibody results were interpreted to be of dubious significance. The correct diagnosis was not made until Borrelia-DNA was detected in the skin biopsy. This is consistent with previous and more recent findings of improved diagnostic accuracy using detection of the Borrelia genome in the skin of patients with acrodermatitis chronica atrophicans, the late cutaneous manifestation of borreliosis $(10,11)$. In addition, our case clearly illustrates that, during treatment with B-cell depleting therapies, infections may give rise to an atypical clinical picture as well as a weak serological response to specific pathogens. Awareness of these circumstances should be highlighted to clinicians serving patients on IMT.

\section{Patient Perspective}

The patient had suffered from knee pain and painful skin erythema for several months before the correct diagnosis was identified. She had been in contact with the primary health care several times before the correct diagnosis was made. Despite the fact that she was treated with IMT, her symptoms were initially interpreted as "non-specific findings of uncertain origin."

\section{DATA AVAILABILITY STATEMENT}

The raw data supporting the conclusions of this article will be made available by the authors, without undue reservation. 


\section{ETHICS STATEMENT}

Ethical review and approval was not required for the study on human participants in accordance with the local legislation and institutional requirements. The patient provided her written informed consent to participate in this study. Written informed consent was obtained from the individual(s) for the publication of any potentially identifiable image or data included in this article.

\section{REFERENCES}

1. Lewandowicz-Uszynska A, Pasternak G, Swierkot J, Bogunia-Kubik K. Primary immunodeficiencies: diseases of children and adults - a review. Adv Exp Med Biol. (2020) 1289:37-54. doi: 10.1007/5584_20 20_556

2. Persson R, Lee S, Ulcickas Yood M, Wagner Usn Mc CM, Minton $\mathrm{N}$, Niemcryk S, et al. Infections in patients diagnosed with multiple sclerosis: a multi-database study. Mult Scler Relat Disord. (2020) 41:101982. doi: 10.1016/j.msard.2020.101982

3. Celius EG. Infections in patients with multiple sclerosis: Implications for disease-modifying therapy. Acta Neurol Scand. (2017) 136(Suppl 201):34-6. doi: 10.1111/ane. 12835

4. Piehl F. A changing treatment landscape for multiple sclerosis: challenges and opportunities. J Intern Med. (2014) 275:364-81. doi: 10.1111/joim.12204

5. Hillert J, Stawiarz L. The Swedish MS registry - clinical support tool and scientific resource. Acta Neurol Scand. (2015) 132:11-9. doi: 10.1111 /ane. 12425

6. Parodis I, Stockfelt M, Sjowall C. B cell therapy in systemic lupus erythematosus: from rationale to clinical practice. Front Med (Lausanne). (2020) 7:316. doi: 10.3389/fmed.2020.00316

7. Luna G, Alping P, Burman J, Fink K, Fogdell-Hahn A, Gunnarsson M, et al. Infection risks among patients with multiple sclerosis treated with fingolimod, natalizumab, rituximab, and injectable therapies. JAMA Neurol. (2020) 77:184-91. doi: 10.1001/jamaneurol.2019.3365

8. Adler S, Krivine A, Weix J, Rozenberg F, Launay O, Huesler J, et al. Protective effect of A/H1N1 vaccination inimmune-mediated disease-a prospectively

\section{AUTHOR CONTRIBUTIONS}

JS, CS, and CD had full access to all of the data and takes responsibility for the integrity, accuracy and interpretation of the data. All authors contributed to the article and approved the submitted version. All authors were involved in drafting the manuscript or revising it critically for important intellectual content and all authors approved the final version to be published.

controlled vaccination study. Rheumatology (Oxford). (2012) 51:695-700. doi: 10.1093/rheumatology/ker389

9. Crnkic Kapetanovic M, Saxne T, Jonsson G, Truedsson L, Geborek P. Rituximab and abatacept but not tocilizumab impair antibody response to pneumococcal conjugate vaccine in patients with rheumatoid arthritis. Arthritis Res Ther. (2013) 15:R171. doi: 10.1186/ar4358

10. von Stedingk LV, Olsson I, Hanson HS, Asbrink E, Hovmark A. Polymerase chain reaction for detection of Borrelia burgdorferi DNA in skin lesions of early and late Lyme borreliosis. Eur J Clin Microbiol Infect Dis. (1995) 14:1-5. doi: 10.1007/BF02112610

11. Lenormand C, Jaulhac B, Debarbieux S, Dupin N, Granel-Brocard F, Adamski $\mathrm{H}$, et al. Expanding the clinicopathological spectrum of late cutaneous Lyme borreliosis (acrodermatitis chronica atrophicans [ACA]): a prospective study of 20 culture- and/or polymerase chain reaction (PCR)-documented cases. $J$ Am Acad Dermatol. (2016) 74:685-92. doi: 10.1016/j.jaad.2015.10.046

Conflict of Interest: The authors declare that the research was conducted in the absence of any commercial or financial relationships that could be construed as a potential conflict of interest.

Copyright (c) 2021 Sjöwall, Xirotagaros, Anderson, Sjöwall and Dahle. This is an open-access article distributed under the terms of the Creative Commons Attribution License (CC BY). The use, distribution or reproduction in other forums is permitted, provided the original author(s) and the copyright owner(s) are credited and that the original publication in this journal is cited, in accordance with accepted academic practice. No use, distribution or reproduction is permitted which does not comply with these terms. 